\title{
VARIAÇÃO SAZONAL DA ESTRUTURA DE MASSAS DE ÁGUA NA PLATAFORMA CONTINENTAL DO AMAZONAS E ÁREA OCEÂNICA ADJACENTE
}

\author{
Alex Costa da Silva ${ }^{1}$, Moacyr Araújo $0^{1,2}$ e Bernard Bourlès ${ }^{3}$ \\ Recebido em 11 março, 2005 / Aceito em 02 setembro, 2005 \\ Received on March 11, 2005 / Accepted on September 02, 2005
}

\begin{abstract}
This work analyzes hydrographic properties (temperature and salinity) obtained from three oceanographic cruises performed along the Amazon continental shelf and adjacent oceanic areas of the western equatorial Atlantic. Field data covered three distinct periods and river discharge situation: the maximum river outflow during boreal spring (May 1999); the transition flow period (maximum to minimum discharges) during summer (August 2001), and minimum river flow during autumn (November 1997). The analysis of CTD hydrographic profiles identified four water masses: Coastal Water (CW), Tropical Surface Water (TSW), South Atlantic Central Water (SACW) and Antarctic Intermediary Water (AIW). Results suggest that wind shear variability acts over the horizontal distribution of surface water masses (CW and TSW), while the subsurface waters (SACW and AIW) are influenced by seasonal variability of the NBC transport and the presence of anticyclonic rings at the NBC retroflection area. Vertical transects analysis show that SACW is strongly expanded during the transition river flow regime (about $70 \mathrm{~m}$ at $49^{\circ} \mathrm{W}$ and $220 \mathrm{~m}$ at $50^{\circ} \mathrm{W}$ ), which corresponds to the period when NBC and North Equatorial Countercurrent (NECC) reach their maximum strengths.
\end{abstract}

Keywords: Amazon shelf, Water masses, North Brazil Current, Program REVIZEE.

RESUMO. Este trabalho analisa as informações de temperatura e salinidade obtidas a partir de três cruzeiros oceanográficos realizados na área da Plataforma Continental do Amazonas e região oceânica adjacente. Os dados foram coletados em diferentes períodos do ano, correspondendo a distintas situações de aporte de águas continentais: mínima descarga do rio Amazonas (outubro/1997), máxima descarga fluvial (maio/1999), e durante o período de transição (máxima para mínima) descarga do rio (agosto/2001). Os resultados permitiram descrever a variação sazonal da distribuição tridimensional das quatro massas de água encontradas: Água Costeira (AC), Água Tropical (AT), Água Central do Atlântico Sul (ACAS) e Água Intermediária da Antártica (AIA). Os resultados sugerem que o cisalhamento eólico age sobre a distribuição temporal das águas superficiais (AC e AT), enquanto que a distribuição das massas d'água de subsuperfície (ACAS e AIA) é influenciada pela variabilidade sazonal da Corrente Norte do Brasil (CNB) e pela presença de vórtices anticiclônicos na região de retroflexão da CNB. Os transectos verticais indicam que a ACAS sofre uma expansão de cerca de $70 \mathrm{~m}\left(49^{\circ} \mathrm{W}\right)$ e de $220 \mathrm{~m}\left(50^{\circ} \mathrm{W}\right)$ durante o período de transição das descargas fluviais, que corresponde à época de máxima ação da CNB e da Contra-corrente Norte Equatorial (CCNE).

Palavras-chave: Plataforma Continental do Amazonas, Massas de água, Corrente Norte do Brasil, Programa REVIZEE.

\footnotetext{
${ }^{1}$ Laboratório de Oceanografia Física Estuarina e Costeira, Departamento de Oceanografia da Universidade Federal de Pernambuco - LOFEC/DOCEAN/UFPE, Av. Arquitetura s/n, 50739-540 Campus Universitário, Recife, PE, Brasil. Tel: +55 (81) 21267224; Fax: +55 (81) 21268225 ou 8227 - E-mail: acostasil@yahoo.com.br; moa@ufpe.br

${ }^{2}$ Bolsista CNPq.

${ }^{3}$ Institut de Rechercher pour le Développement - IRD, Boite Postale 70, 29280, Plouzané, France. Phone: +33.2.98224665; Fax: +33.2.98224514 - E-mail: bernard.bourles@ird.fr
} 


\section{INTRODUÇÃo}

A área do oceano Atlântico equatorial adjacente à Região Norte do Brasil é identificada como um sistema marinho de alta energia, resultado da ação combinada da corrente de fronteira oeste, dos ventos alísios, das oscilações de marés, e do lançamento de águas continentais oriundas do rio Pará e sobretudo do rio Amazonas. Este último descarrega em torno de $180.000 \mathrm{~m}^{3} \mathrm{~s}^{-1}$ de água doce no Oceano Atlântico, contribuindo com cerca de 18\% do total de água doce continental lançada nos oceanos (Oltman, 1968; Muller-Karger et al., 1988). Essa vazão varia sazonalmente, com máximo em torno de $220.000 \mathrm{~m}^{3} \mathrm{~s}^{-1}$ em maio, e mínimo em torno de $100.000 \mathrm{~m}^{3} \mathrm{~s}^{-1}$ em novembro (Geyer et al., 1996). A água doce proveniente do rio Amazonas forma uma pluma superficial que pode exceder mais de $200 \mathrm{~km}$ oceano adentro e cerca de 1000 km em direção noroeste (Gibbs, 1970; Lentz \& Limeburner, 1995).

Além da grande quantidade de movimento resultante do aporte continental, a área da Plataforma Continental do Amazonas (referenciada a seguir por PCA) e região oceânica adjacente, encontra-se sob influência direta da Corrente Norte do Brasil (CNB). Segundo Richardson et al. (1994), a CNB flui ao longo da quebra da Plataforma do Amazonas, sobre o talude, predominantemente no sentido noroeste, com velocidades entre 0,5-1,0 $\mathrm{m} \mathrm{s}^{-1}$. Johns et al. (1998) estimaram valores de transporte da CNB adjacente à costa amazônica $\left(4^{\circ} \mathrm{N}-45^{\circ} \mathrm{W}\right)$ variando entre 35 Sv $\left(1 \mathrm{~Sv}=10^{6} \mathrm{~m}^{3} \mathrm{~s}^{-1}\right)$ nos meses de julho-agosto e $13 \mathrm{~Sv}$ em abril-maio. Mais ao norte, nas proximidades do estado do Amapá, esta corrente de fronteira sofre sazonalmente uma retroflexão para leste, alimentando a Contra-Corrente Norte Equatorial (CCNE), e contribuindo para formação de vórtices anticiclônicos (Johns et al., 1990; Didden \& Schott, 1993; Fratantoni et al., 1995; Schott et al., 1998).

Outro fator importante na dinâmica da região de estudo é associado à variabilidade sazonal do cisalhamento eólico, que transfere distintamente quantidade de movimento da atmosfera para 0 oceano em função da época do ano. Tratam-se dos ventos alísios, que apresentam uma variação sazonal vinculada às oscilações da Zona de Convergência Inter-Tropical Atmosférica (ZCIT). A ZCIT se localiza predominantemente no hemisfério norte, migrando periodicamente das proximidades do equador em março-abril, para cerca de $5^{\circ} \mathrm{N}$, em agosto-setembro (Castro \& Miranda, 1998). Esta variação sazonal da zona de convergência faz com que os ventos alísios, atuantes na PCA e área oceânica adjacente, sejam predominantemente de sudeste no período de junho a novembro, e de nordeste nos meses de dezembro a maio
(Nittrouer \& Demaster, 1996; Geyer et al., 1996).

Este trabalho tem por objetivo analisar a variabilidade sazonal das distribuições espaciais das massas de água existentes na PCA e área oceânica adjacente, durante os períodos de descarga máxima do rio Amazonas (maio de 1999), de transição (agosto de 2001), e durante a época de mínima descarga fluvial (outubro de 1997), identificando as forçantes geofísicas potencialmente determinantes destas variabilidades. Este trabalho é um dos produtos cientííicos do Programa REVIZEE (Programa Nacional de Avaliação do Potencial Sustentável de Recursos Vivos da Zona Econômica Exclusiva).

\section{METODOLOGIA Área de estudo}

A área de estudo compreeende a seção do oceano Atlântico equatorial oeste limitada pelas coordenadas Lat. $2,5^{\circ} \mathrm{S}-7^{\circ} \mathrm{N}$ e Long. $46^{\circ} \mathrm{W}-51^{\circ} \mathrm{W}$. Parte dessa região corresponde à PCA, que se estende desde a foz do rio Pará (PA) até o Cabo Norte (AP). A isóbata de $100 \mathrm{~m}$ marca a quebra da plataforma continental (Nittrouer \& Demaster, 1986). Na frente da foz do rio Amazonas, a PCA atinge uma largura de $330 \mathrm{~km}$, diminuindo em direção ao Cabo Orange (Flood \& Damuth, 1987).

A área de estudo é representada na Figura 1, onde são indicadas as localizações das estações de coleta (CTD), e dos transectos verticais Perfil (A/S) e Perfil (W), perpendiculares à costa. A escolha da localização do Perfil (A/S) para análise nesse trabaIho teve como objetivo comparar as características termohalinas das massas de água com as medidas de corrente dos Projetos AMASSEDS (A Multidisciplinary Amazon Shelf Sediment Study) e STACS (Subtropical Atlantic Climate Studies), obtidas através de fundeios e de perfilagens de ADCP (Acoustic Doppler Current Profiler) realizadas nos anos 1989-1991 ao longo de um transecto muito próximo do Perfil (A/S) escolhido (Fig. 1, de Johns et al., 1998). Já o Perfil (W), situado na altura do Cabo Orande, Amapá (Figura 1), foi definido com o intuito de também possibilitar a comparação das informações do Programa REVIZEE com as perfilagens de ADCP/Pegasus realizadas na região de retroflexão da CNB por Wilson et al. (1994) e Brown et al. (1992), este último no âmbito do Projeto WESTRAX (Western Tropical Atlantic Experiment)(Fig. 2, de Bourlès et al., 1999).

\section{Base de dados}

Os dados utilizados neste trabalho foram coletados durante três cruzeiros oceanográficos realizados pelo NOc. Antares na região Norte do Brasil, como parte das atividades do Programa REVIZEE 


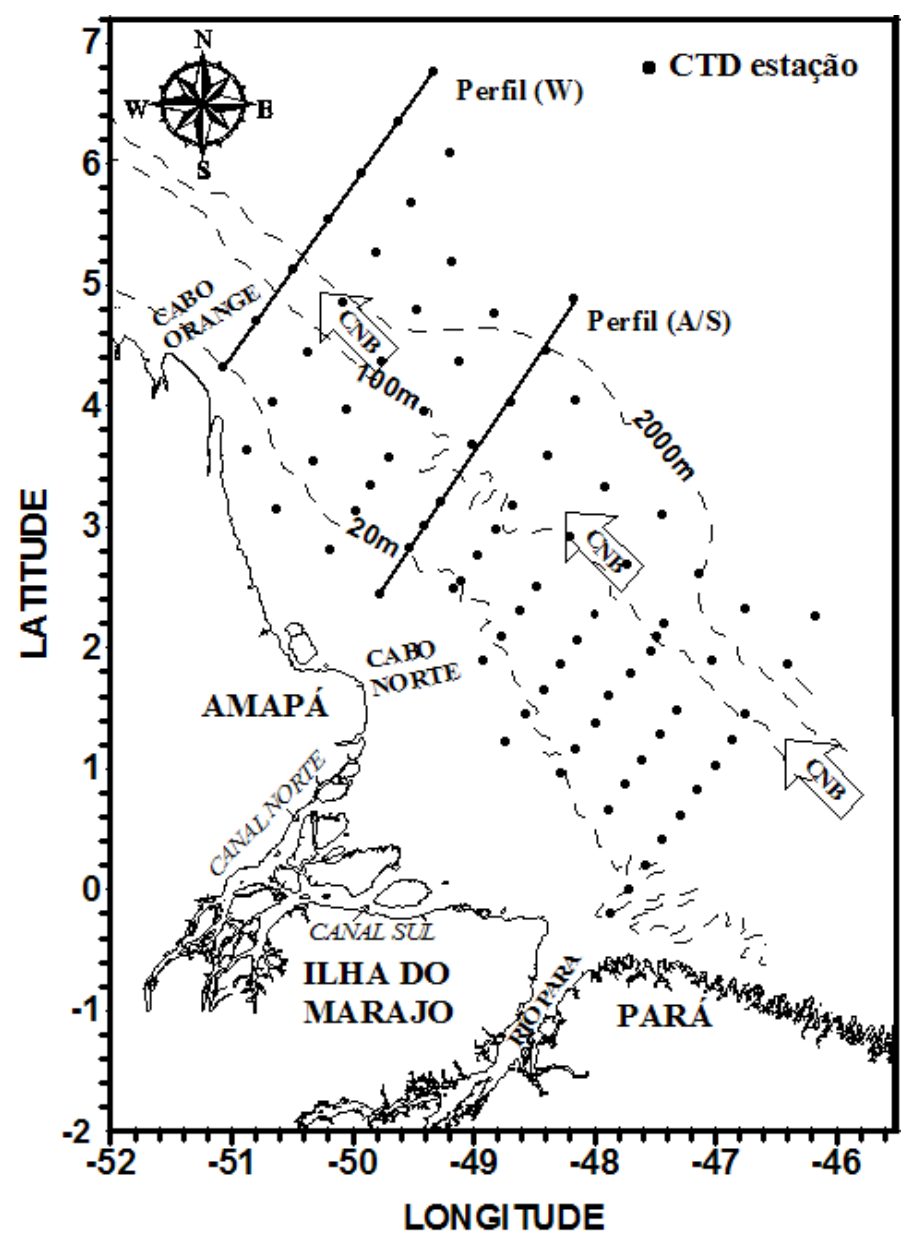

Figura 1 - Mapa de localização da área de estudo, indicando as estações de coleta (CTD), os transectos verticais Perfis (A/S) e (W), e a Corrente Norte do Brasil (CNB), fluindo para noroeste ao longo da margem continental amazônica.

(Programa Nacional de Avaliação do Potencial Sustentável de Recursos Vivos da Zona Econômica Exclusiva). 0 Programa REVIZEE, sob coordenação da Comissão Interministerial de Recursos do Mar (CIRM), é resultado do compromisso que o Brasil assumiu perante à Organização das Nações Unidas (ONU), ao ratificar em 1988 a Convenção Internacional da Lei do Mar, em validade desde 1994. 0 objetivo principal do Programa REVIZEE foi realizar um levantamento dos recursos vivos (e dos fatores abióticos correlacionados) presentes na Zona Econômica Exclusiva brasileira.

0 conjunto de dados analisados correspondem a 248 perfis verticais contínuos de temperatura e salinidade obtidos com equipamento CTD Sea Bird Electronics SBE911p/us, equipado com bomba centrífuga e sensores de alta resolução para medições de condutividade (resolução $=0,00004 \mathrm{~S} \mathrm{~m}^{-1}$ ), temperatura (resolução $=0,0003^{\circ} \mathrm{C}$ ) e pressão (resolução $=0,068 \mathrm{db}$ ). A salinidade foi calculada com os algoritmos da escala prática (PSS178), adotada pela UNESCO, em função da razão de condutividade elétrica, temperatura e pressão (UNESCO, 1981).

Durante todos os cruzeiros o CTD foi operado até cerca de $1000 \mathrm{~m}$ de profundidade, com velocidade descendente de $1 \mathrm{~m}$ $\mathrm{s}^{-1}$, e uma freqüência de amostragem de $24 \mathrm{~Hz}$. Durante as perfilagens o equipamento foi mantido conectado a uma plataforma de bordo SBE 11p/us, o que permitiu um acompanhamento em tempo real da aquisição e primeira verificação da qualidade dos dados. A Tabela 1 traz uma síntese dos dados utilizados neste trabalho.

Os três cruzeiros oceanográficos foram realizados épocas diferentes do ano, associadas às distintas situações de aporte continental de água doce, ou seja: durante o período de descarga 
Tabela 1 - Síntese das perfilagens de CTD obtidas durante as expedições oceanográficas do Programa REVIZEE, Região Norte, utilizadas neste trabalho.

\begin{tabular}{|c|c|c|}
\hline Hidrologia do rio Amazonas & Período & Qde. perfis de CTD \\
\hline Descarga mínima & Outubro de 1997 & 90 \\
\hline Descarga máxima & Maio de 1999 & 87 \\
\hline Transição máxima-mínima descarga & Agosto de 2001 & 71 \\
\hline
\end{tabular}

mínima do rio Amazonas (outubro de 1997), durante a descarga máxima do rio Amazonas (maio de 1999), e durante o período de transição de máxima para mínima descarga (agosto de 2001).

\section{RESULTADOS}

\section{Diagrama T-S e massas de água}

Baseado nas propriedades observadas durante as campanhas de outubro de 1997, maio de 1999 e agosto de 2001, foi possível identificar quatro massas de água na região estudada de acordo com os critérios apresentados na Tabela 2. As distribuições espaciais entre estas massas de água foram identificadas a partir da localização tridimensional dos respectivos valores de limite isopicnais $\left(\sigma_{\theta}\right)$. Neste caso, a isopicna $\sigma_{\theta} \leq 21,6$ registra 0 limite de influência da AC; 0 limite entre AT-ACAS é marcado pela $\sigma_{\theta}=24,5$; e o limite entre ACAS-AIA é associado à $\sigma_{\theta}=27,1$. Estes valores limites foram definidas a partir dos estudos da DHN (1989), Stramma \& Schott (1999) e Barnier et al. (2001).

As massas de água são definidas pelo diagrama T-S, gerado a partir da correlação entre os valores de temperatura (T) e salinidade (S). A Figura 2 apresenta o diagrama T-S obtido a partir dos valores de temperatura e salinidade medidos ao longo das colunas d'água das estações de amostragem do Perfil A (Figura 1).

A Água Costeira (AC) na região de estudo é identificada pelo seu alto valor de temperatura e baixo valor de salinidade, como resultado do aporte de água doce proveniente do rio Amazonas (Curtin, 1986; DHN, 1989). Na área oceânica, da superfície para o fundo, a primeira massa d' água encontrada na região é a Água Tropical (AT). Abaixo desta registra-se a presença da Água Central do Atlântico Sul (ACAS), transportada para a região de estudo pela CNB e Sub-Corrente Norte do Brasil (SCNB). A ACAS apresenta baixo valor de salinidade e alto valor de oxigênio dissolvido. No intervalo de $500 \mathrm{~m}$ a cerca de $1200 \mathrm{~m}$ de profundidade encontrase a Água Intermediária da Antártica (AIA), com baixo valor de temperatura (Stramma \& Schott, 1999; Barnier et al. 2001).

\section{Estrutura vertical das massas de água}

Nesta seção são apresentadas as distribuições horizontais das profundidades limites entre as massas de água (AC-AT), (ATACAS) e (ACAS-AIA) na região de estudo. Estas profundidades foram obtidas considerando os respectivos limites isopicnais entre as massas d'água, conforme descrito na seção anterior. A Figura 3 apresenta as profundidades limites entre a AC e a AT (profundidade do topo da AT), para os três diferentes períodos de campanha. Durante todos os cruzeiros foi confirmada a influência de uma pluma de água de baixo valor de salinidade provinda do rio Amazonas, que se estende sobre a PCA, conforme evidenciado por Lentz \& Limeburner (1995) e Curtin (1986). Na região que sofre influência dessa pluma ocorre 0 afundamento da AT. Durante os meses de agosto de 2001 e de outubro de 1997 (períodos de transição e de baixa descarga do rio Amazonas) (Figuras 3(A) e (B)), a profundidade do topo da AT sofre um ligeiro afundamento na região noroeste da plataforma amazônica, que nesta ocasião se encontra sobre influência simultânea da CNB e dos ventos alísios de sudeste. Já no mês de maio de 1999 (período de máxima descarga do rio Amazonas) (Figura 3(A)), constata-se que a água doce provinda do rio Amazonas fica confinada na área frontal à sua foz, ocorrendo um afundamento significativo do topo da AT na PCA e na área oceânica adjacente, alcançando profundidades superiores a $25 \mathrm{~m}$. Neste período do ano os ventos alísios de nordeste incidem quase que perpendicularmente às isóbatas, pressionando a pluma para junto da costa, e reduzindo assim sua expansão lateral.

A distribuição horizontal das profundidades que limitam a ATACAS nos três períodos estudados é apresentada na Figura 4. Estas distribuições demostraram uma elevação do topo da ACAS sobre a quebra da PCA (isóbata de $100 \mathrm{~m}$ ). Durante 0 mês de maio de 1999 (Figura 4(A)), 0 topo da ACAS se encontra a uma profundidade de cerca de $90 \mathrm{~m}$ ao longo da quebra da plataforma, chegando a atingir 70 metros no talude em frente do Cabo Norte, no estado do Amapá. Durante os meses de agosto de 2001 e de outubro de 1997 (Figura 4(B) e (C), respectivamente), observase igualmente uma resuspensão da ACAS na região da quebra 
Tabela 2 - Valores de temperatura $\left({ }^{\circ} \mathrm{C}\right)$ e salinidade (ups) característicos das respectivas massas de água observadas na área de estudo.

\begin{tabular}{|c|c|c|}
\hline Massa d'água & Temperatura $\left({ }^{\circ} \mathrm{C}\right)$ & Salinidade \\
\hline Água Costeira (AC) & $26,00-28,87$ & $\leq 34,00$ \\
\hline Água Tropical (AT) & $>18,00$ & $>36,00$ \\
\hline Água Central do Atlantico Sul (ACAS) & $5,95-18,35$ & $34,52-36,40$ \\
\hline Água Intermediaria da Antartica (AIA) & $4,92-5,90$ & $34,48-34,78$ \\
\hline
\end{tabular}

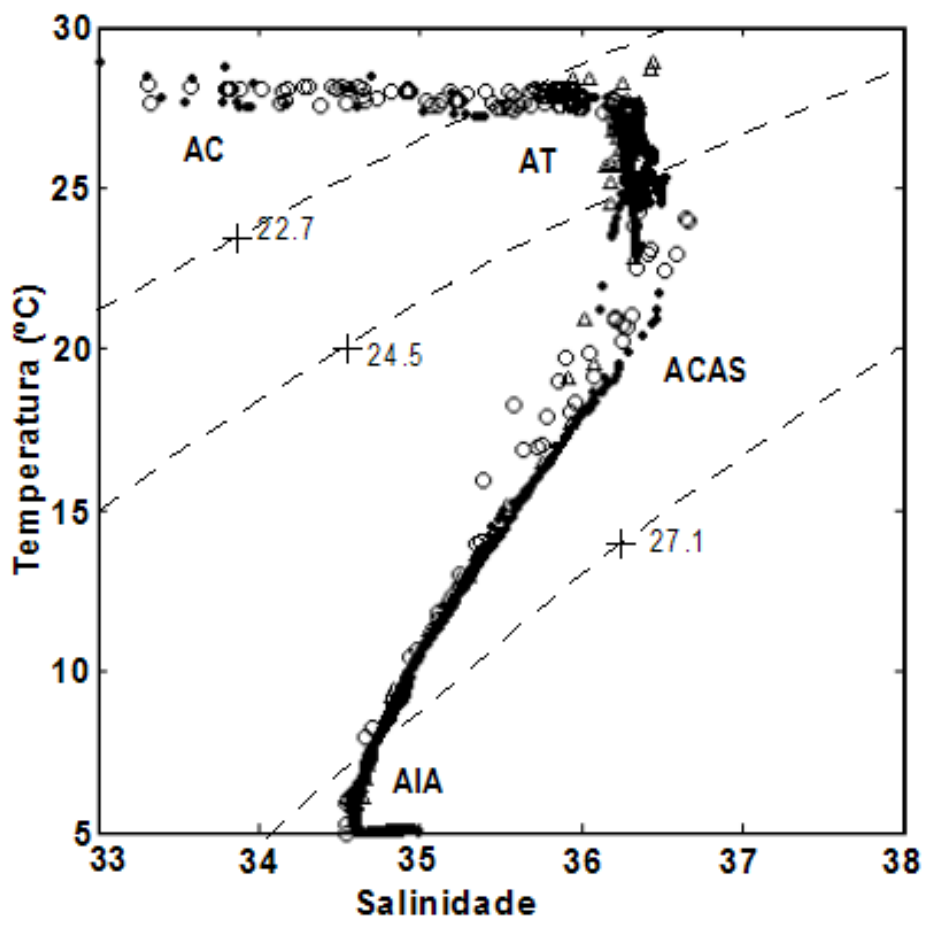

Figura 2 - Diagrama T-S realizado a partir dos dados coletados ao longo do Perfil (A/S) durante o período de descarga máxima (0), período de transição de descarga máxima para mínima (•), e durante o período de descarga mínima $(\Delta)$. No diagrama são identificadas as quatro massas de água da região, com seus respectivos limites isopicnais $\left(\sigma_{\theta}\right): \sigma_{\theta} \leq 21,6$ registra o limite de influência da AC; 0 limite entre AT e ACAS é marcado pela isopicna $\sigma_{\theta}=24,5$; e 0 limite ACAS-AIA é definido pela isopicna $\sigma_{\theta}=27,1$.

da PCA, principalmente na direção do Cabo Norte e a noroeste deste, tendendo a aumentar sua profundidade com 0 afastamento da costa. Para estes dois períodos, entretanto, as isóbatas de profundidade limite da AT-ACAS encontram-se mais alinhadas com as isóbatas da PCA, o que não ocorreu durante o período de vazão máxima do rio.

As distribuições horizontais dos limites de profundidade entre as massas de água ACAS e AIA apresentaram durante o mês de outubro de 1997 (Figura 5(C)) uma elevação do topo da AIA $(\approx 400 \mathrm{~m}$ ) próximo à foz do rio e a noroeste desta, e um afundamento desta massa de água na área frontal ao cabo Norte
( $\approx 500 \mathrm{~m}$ ). Durante o mês de maio de 1999 (Figura 5 (A)), o topo da AIA também apresentou uma elevação na direção da foz do rio e na região que compreende a quebra da PCA, afundando à medida que aumenta a distância da costa. Durante o mês de agosto de 2001 (Figura 5(B)), verifica-se um afundamento no topo da AIA $(\approx 540 \mathrm{~m})$ em toda região estudada.

\section{DISCUSSÃO}

A região equatorial oeste do oceano Atlântico vem atraindo uma quantidade significativa de pesquisas científicas, sobretudo nos últimos vinte anos. Em particular, o elevado aporte continen- 

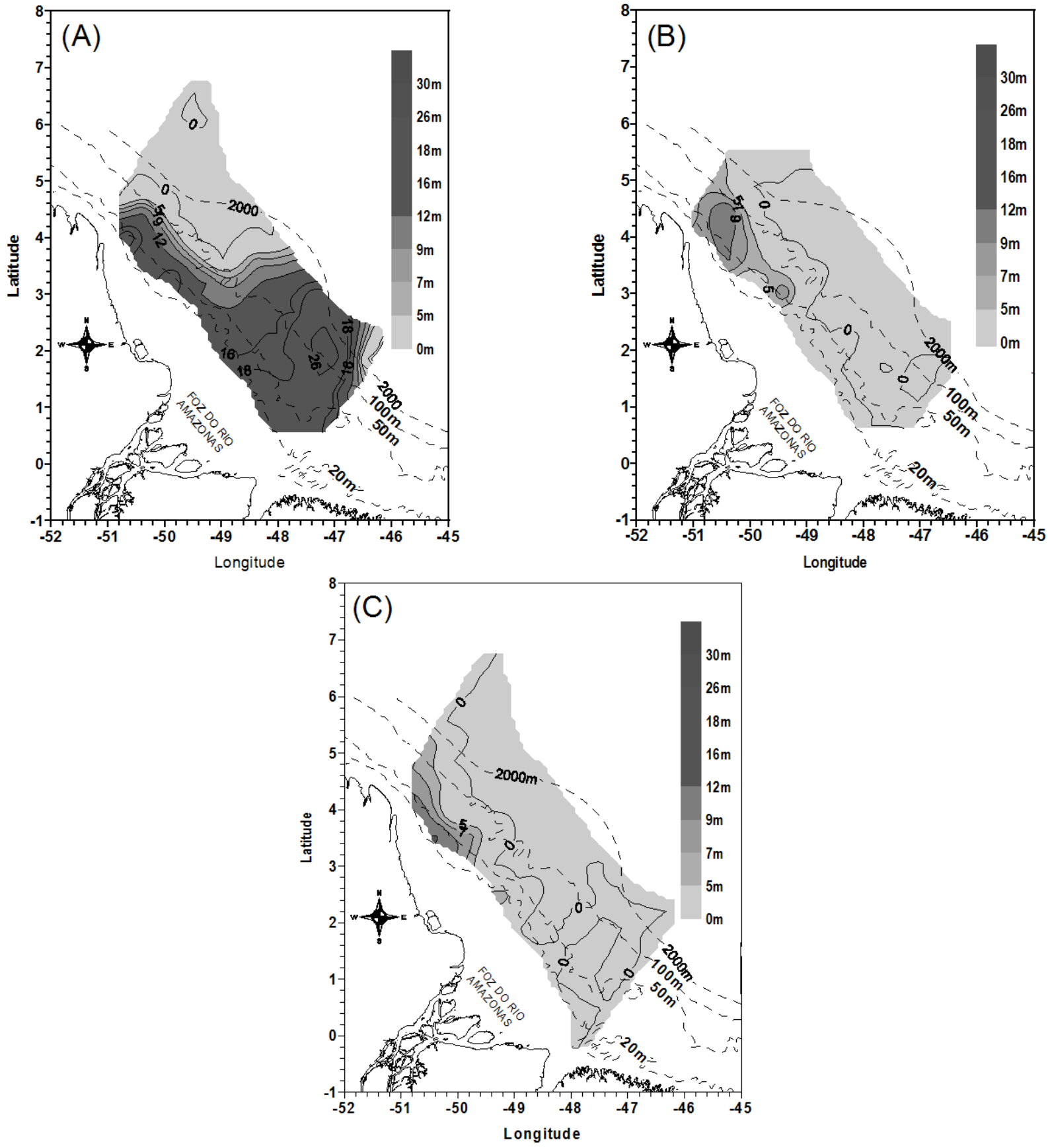

Figura 3 - Distribuição horizontal das profundidades limites entre a AC e a AT na PCA e região oceânica adjacente durante: (A) período de descarga máxima do rio Amazonas (maio de 1999); (B) período de transição entre descarga máxima para mínima do rio (agosto de 2001); e (C) período de descarga mínima do rio Amazonas (outubro de 1997).

tal de águas doces (rios Amazonas e Pará, entre outros), aliada à presença de fortes correntes de fronteira que são modificadas sazonalmente pela variabilidade do cisalhamento eólico (proximidade da Zona de Convergência Intertropical), transfor- mam a região costeira e oceânica adjacente à costa Norte do Brasil num local privilegiado, para onde convergem as águas superficias quentes do sul e as águas frias profundas do Atlântico norte. Uma análise objetiva dos principais trabalhos desenvolvidos na 

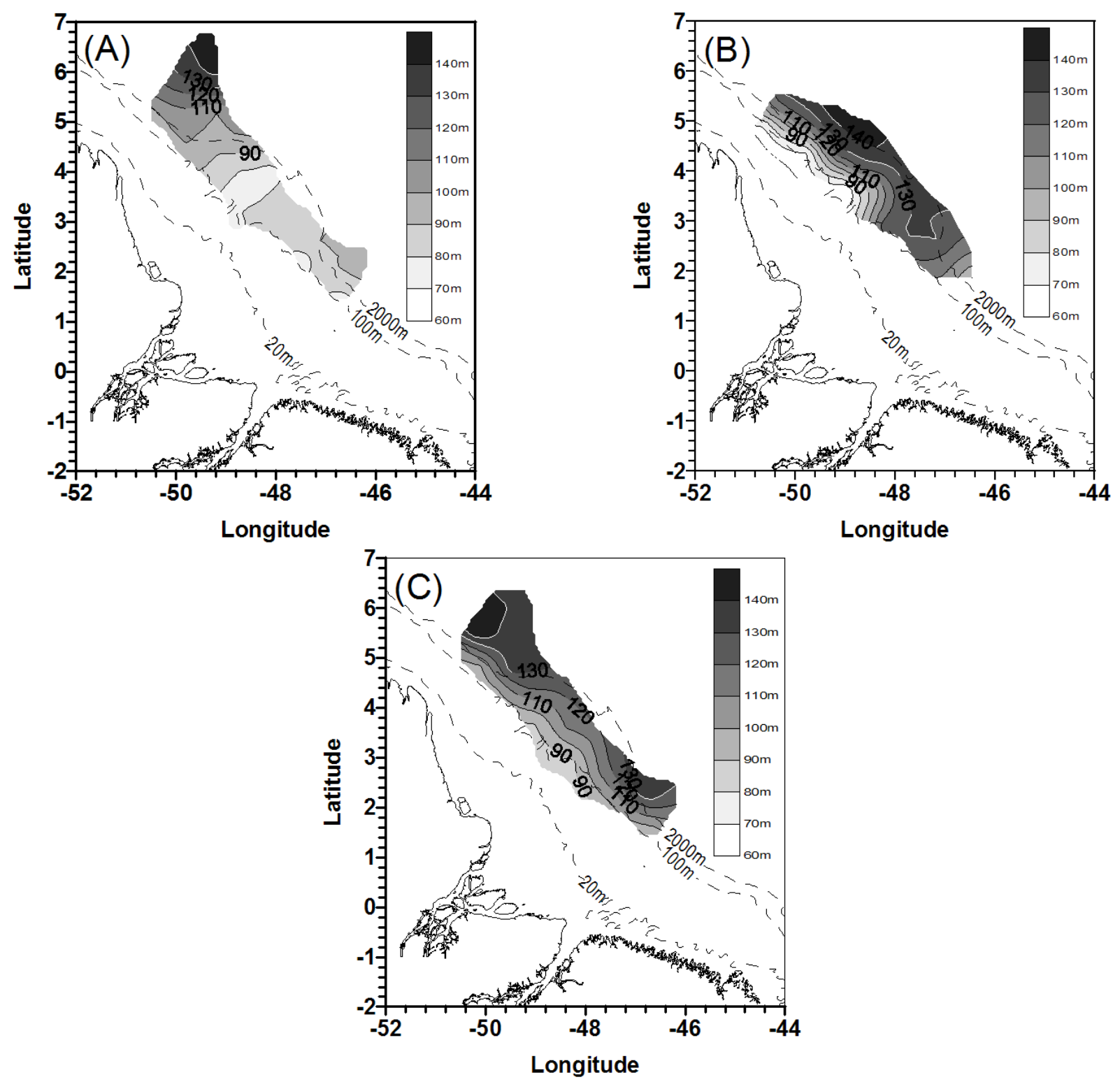

Figura 4 - Distribuição horizontal das profundidades limites entre a AT e a ACAS na PCA e região oceânica adjacente durante: (A) período de descarga máxima do rio Amazonas (maio de 1999); (B) período de transição entre descarga máxima para mínima do rio (agosto de 2001); e (C) período de descarga mínima do rio Amazonas (outubro de 1997).

região permite classificá-los de acordo com a área de atuação. Num primeiro conjunto, estão os estudos que se concentraram na região da Plataforma Continental Amazônica (limitados à quebra da PCA, isóbata $100 \mathrm{~m}$ ), que podem ser representados pelos experimentos do Projeto AMASSEDS. Estes esforços possibilitaram uma descrição mais detalhada das massas de água e da dinâmica das correntes de maré sobre a PCA.

0 segundo conjunto de trabalhos desenvolvidos na região oeste do Atlântico equatorial se limitara quase que exclusiva- mente à caracterização da região oceânica adjacente à PCA, na grande maioria das vezes além da isóbata de $1000 \mathrm{~m}$. Assim foram os casos das expedições e experimentos realizados no âmbito dos Projetos STACS (Johns et al., 1998), Westrax (Brown et al., 1992), e mais recentemente pelo Projeto North Brazil Current Rings Experiment (Garzoli et al., 2004). Estes trabalhos trouxeram informações até então desconhecidas acerca da variabilidade espaço-temporal da CNB, de sua ligação com a Contra-corrente Norte Equatorial, e do papel dos vórtices anticiclônicos gerados 

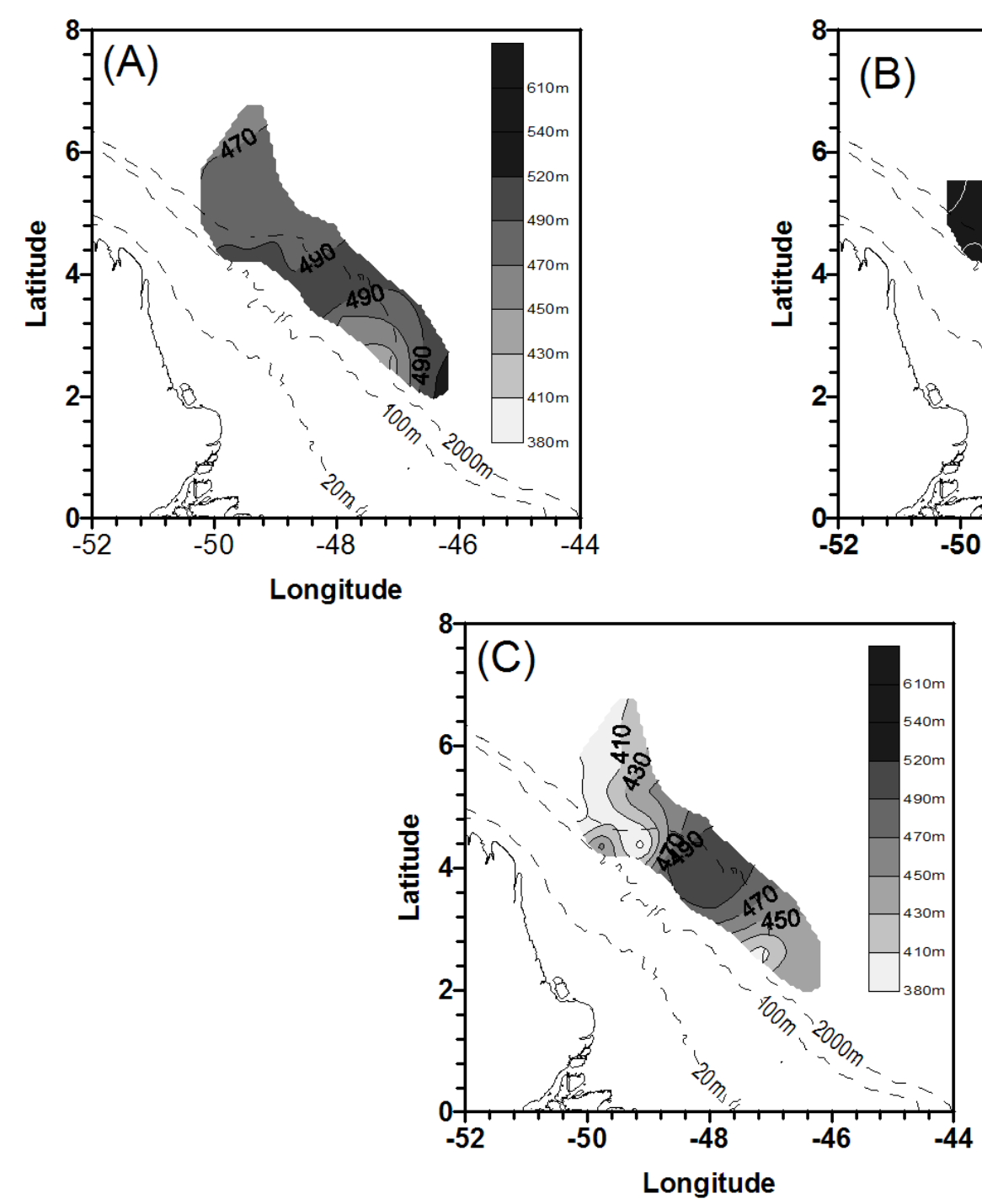

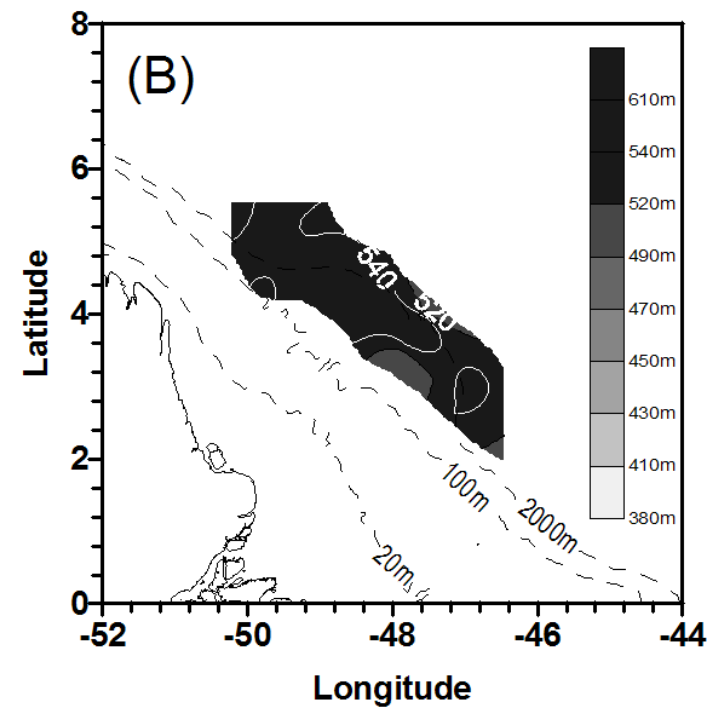

Longitude do rio Amazonas (maio de 1999); (B) período de transição entre descarga máxima para mínima do rio (agosto de 2001); e (C) período de descarga mínima do rio Amazonas (outubro de 1997).

na região de retroflexão da CNB.

Entre a quebra da PCA e a isóbata de $1000 \mathrm{~m}$, entretanto, pouca informação existe. Esta área, que engloba 0 talude da PCA, é um local-chave para compreensão dos mecanismos de interação entre a plataforma continental a área oceânica adjacente. Visando analisar o domínio e a variabilidade espacial das massas de água nesta região, foram traçados dois transectos verticais perpendiculares à costa, para os meses de maio de 1999, agosto de 2001 e outubro de 1997 (períodos de descarga máxima, transição e mínima do rio Amazonas) (Figura 1). A Figura 6 corresponde ao Perfil (A/S) e a Figura 7 representa o Perfil (W).
Os resultados para o transecto Perfil (A/S) demostram que, durante todos os períodos de coleta, foi registrada uma massa de Água Costeira (AC) de baixo valor de salinidade $(\leq 34)$ e alto valor de temperatura, dando origem à formação de haloclina que se situou em torno de $10 \mathrm{~m}$ a $20 \mathrm{~m}$ de profundidade (Figura 6).

No mês de maio de 1999 (Figura 6(A)) observa-se a presença superficial da AC até cerca de $310 \mathrm{~km}$ de distância da costa. Nesta época do ano, a ação dos ventos alísios de nordeste minimizam 0 transporte da pluma de água doce para noroeste. Durante o mês de agosto de 2001 (Figura 6(B)), foi observada a presença de AC até aproximadamente $212 \mathrm{~km}$ de distância da costa, enquanto que 

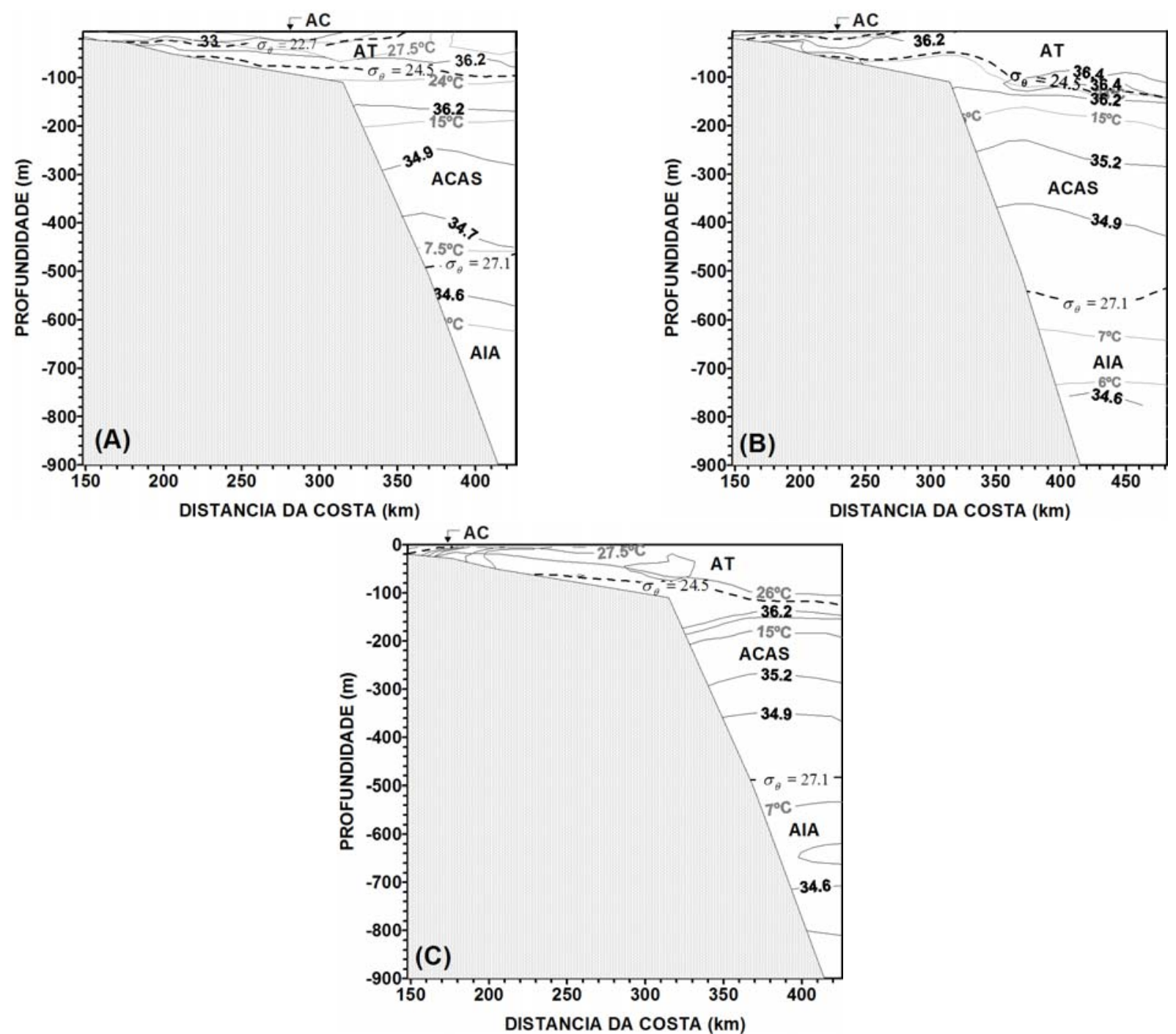

Figura 6 - Distribuição vertical das massas de água ao longo do transecto Perfil (A/S) durante: (A) período de descarga máxima do rio Amazonas (maio de 1999); (B) período de transição entre descarga máxima para mínima do rio (agosto de 2001); e (C) período de descarga mínima do rio Amazonas (outubro de 1997).

no mês de outubro de 1997 (Figura 6(C)) a pluma atingiu 140 km de distância da costa. A ação dos ventos alísios de sudeste, característicos destas épocas do ano, facilitam o transporte da pluma para noroeste, distribuindo-a mais homogeneamente ao longo de toda a costa amazônica.

Os gráficos da Figura 6 indicam que a AT esteve presente abaixo da AC na área da PCA, e na superfície nas regiões que não sofrem influência das águas do rio Amazonas. Abaixo da AT, até a profundidade de aproximadamente $490 \mathrm{~m}$ a $550 \mathrm{~m}$, foi identificada a presença da ACAS, e sob esta, a AIA (Figura 6). Durante o mês de maio de 1999 (Figura 6(A)), o topo da ACAS foi registrado na profundidade de $70 \mathrm{~m}$ ao longo do Perfil A. No mês de agosto de 2001 (Figura 6(B)), observa-se uma elevação do topo da ACAS, invandido a plataforma (chegando a atingir uma profundidade de $50 \mathrm{~m}$ ), e em seguida aumentando sua profundidade com 0 afastamento da costa. Durante o mês de outubro de 1997 (Figura 6(C)), o topo da ACAS também apresenta uma elevação próximo à da quebra do talude da plataforma (isóbata $100 \mathrm{~m}$ ), atingindo uma profundidade de 80 metros. Assim como para as outras épocas do ano, observa-se o neste período um aumento desta profundidade à medida que se adentra no oceano. Nos meses de maio de 1999 e outubro de 1997, o topo da AIA no Perfil A se localizou a uma profundidade de cerca de $490 \mathrm{~m}$. No mês de agosto de 2001 este sofreu um maior rebaixamento atingindo 
a profundidade de $550 \mathrm{~m}$ (Figura 6)

Ainda com relação ao Perfil (A/S), um conjunto significativo de medidas de corrente foi obtida no âmbito dos Projetos AMASSEDS (A Multidisciplinary Amazon Shelf Sediment Study)e STACS (Subtropical Atlantic Climate Studies)(Johns et al., 1998). Estas medições foram realizadas exatamente ao longo de um transecto perpendicular à costa (muito próximo do Perfil (A/S), Figura 1). Os resultados destas análises indicaram que a CNB possui uma grande variabilidade sazonal nesta latitude. Durante os meses de abril-maio, que engloba a época de descarga máxima do rio Amazonas, verificou-se que a CNB se mantem praticamente estreita e confinada à região de talude da PCA, transportando cerca de $13 \mathrm{~Sv}$ (menor valor anual). Já durante os meses de julhoagosto, época que coincide com o período de transição entre as maiores e menores vazões do rio, a CNB se transforma numa corrente de fronteira mais larga e mais profunda, alcançando valores de transporte da ordem de $36 \mathrm{~Sv}$ (máximo transporte anual) (Johns et al., 1998). Os dados do Programa REVIZEE indicam, para este último período, uma expansão da ACAS (Figura 6(B)), quando comparado ao mês de maio de 1999 (Figura 6(A)). Esta expansão da ACAS, traduzida por uma elevação relativa de seu topo e afundamento relativo de sua base (que corresponde ao topo da AIA), coincide, portanto, com o período de máximo transporte da CNB naquela latitude.

Os transectos apresentados na Figura 7 foram traçados a partir dos dados do Programa REVIZEE, obtidos ao longo do Perfil (W) (Figura 1). A distribuição espacial das massas de água nesta região diferem significativamente daquela observada no Perfil (A/S), Figura 6. Analisando-se inicialmente as águas superficiais, observa-se que a pluma de massa de água costeira fica restrita a cerca de $140 \mathrm{~km}$ de distância da costa no mês de maio de 1999 (Figura 7(A)), expandindo-se consideravelmente oceano adentro (270 km) no mês de agosto de 2001 (Figura 7(B)). Estas distribuiç̧ões são qualitativamente opostas àquelas verificadas nos transectos do Perfil (A/S), situado mais próximo à foz do rio Amazonas, quando se obteve, inversamente, uma maior penetração oceânica da pluma de água doce no período de descarga máxima (Figura 6(A)), e um menor alcance da pluma durante a época de transição das vazões (Figura 6(B)).

A Água Tropical ao longo do Perfil (W) esteve presente abaixo da AC na região da PCA. Abaixo desta zona, até profundidades de aproximadamente $400 \mathrm{~m}$ a $620 \mathrm{~m}$ (em função da época do ano), foi igualmente constatada a presença da ACAS, e abaixo desta, a AIA (Figura 7). No que se refere à sazonalidade, verifica-se um comportamento aproximadamente semelhante com relação à localização do topo da ACAS nas três épocas do ano. Esta situou- se a cerca de $70 \mathrm{~m}$ nas proximidades de quebra da PCA, sofrendo um rebaixamento com 0 afastamento da costa (até aproximadamente 140 m na área oceânica).

Situações nitidamente distintas, entretanto, são observadas quando se comparam as profundidades limite entre as massas de água ACAS e AIA ao longo do Perfil (W). Neste caso, durante os meses de outubro de 1997 (Figura 7(C)) e de maio de 1999 (Figura 7(A)), o topo da AIA na região do Perfil (W) se localizou entre $400 \mathrm{~m}$ e $450 \mathrm{~m}$, respectivamente. Já para 0 mês de agosto de 2001, verifica-se que houve um rebaixamento significativo do limite ACAS-AIA, que atingem uma profundidade de $620 \mathrm{~m}$ (Figura 7(B)). Este cenário caracteriza uma expansão considerável do domínio das águas centrais no mês de agosto, quando comparado aos outros períodos analisados, o que certamente tem relação com a dinâmica das correntes de fronteira existentes na região. As análises de Bourlès et al. (1999), realizadas a partir das perfilagens de ADCP e correntometria Pegasus (Wilson et al., 1994; Brown et al., 1992), por exemplo, indicam que os períodos de agosto e setembro se caraterizam por intensa atividade de formação de vórtices anticiclônicos subsuperficiais na área do Perfil (W). Mais recentemente, as medidas de ADCP/Ecosonda invertida analisadas por Garzoli et al. (2004), no âmbito do Projeto North Brazil Current Rings Experiment (19992000), confirmam ser 0 mês de setembro o período de máximo transporte para sudeste das águas da CNB que são retroflectadas na costa amazônica. Esta época do ano coincide com o período de máxima ação da Contra-corrente Norte Equatorial (CCNE), que é em parte alimentada pela retroflexão da CNB. Esta intensa atividade, por exemplo, não é, nem verificada nos meses de marçoabril, período em que a CCNE não foi observada na região estudada (Garzoli \& Katz, 1983), nem durante o mês de maio, quando a intensidade da CNB é mínima (Johns et al., 1998; Garzoli et al., 2004). Em sua análise, Garzoli et al. (2004) sugerem ainda que a ocorrência quase que simultânea dos picos do transporte pela CNB e de transporte de volume d'água retroflectado pela CCNE, favorecem a formação de vórtices anticiclônicos, gerando um maior deslocamento da CNB no sentido noroeste.

\section{CONCLUSÕES}

Este trabalho analisa informações hidrográficas obtidas na região da Zona Econômica Exclusiva brasileira compreendendo a Plataforma Continental Amazônica área oceânica adjacente. Trata-se de uma contribuição ao conhecimento da variação sazonal da estrutura termohalina numa porção do oceano ainda pouco pesquisada. Expedições e experimentos anteriores se restringiram, seja 

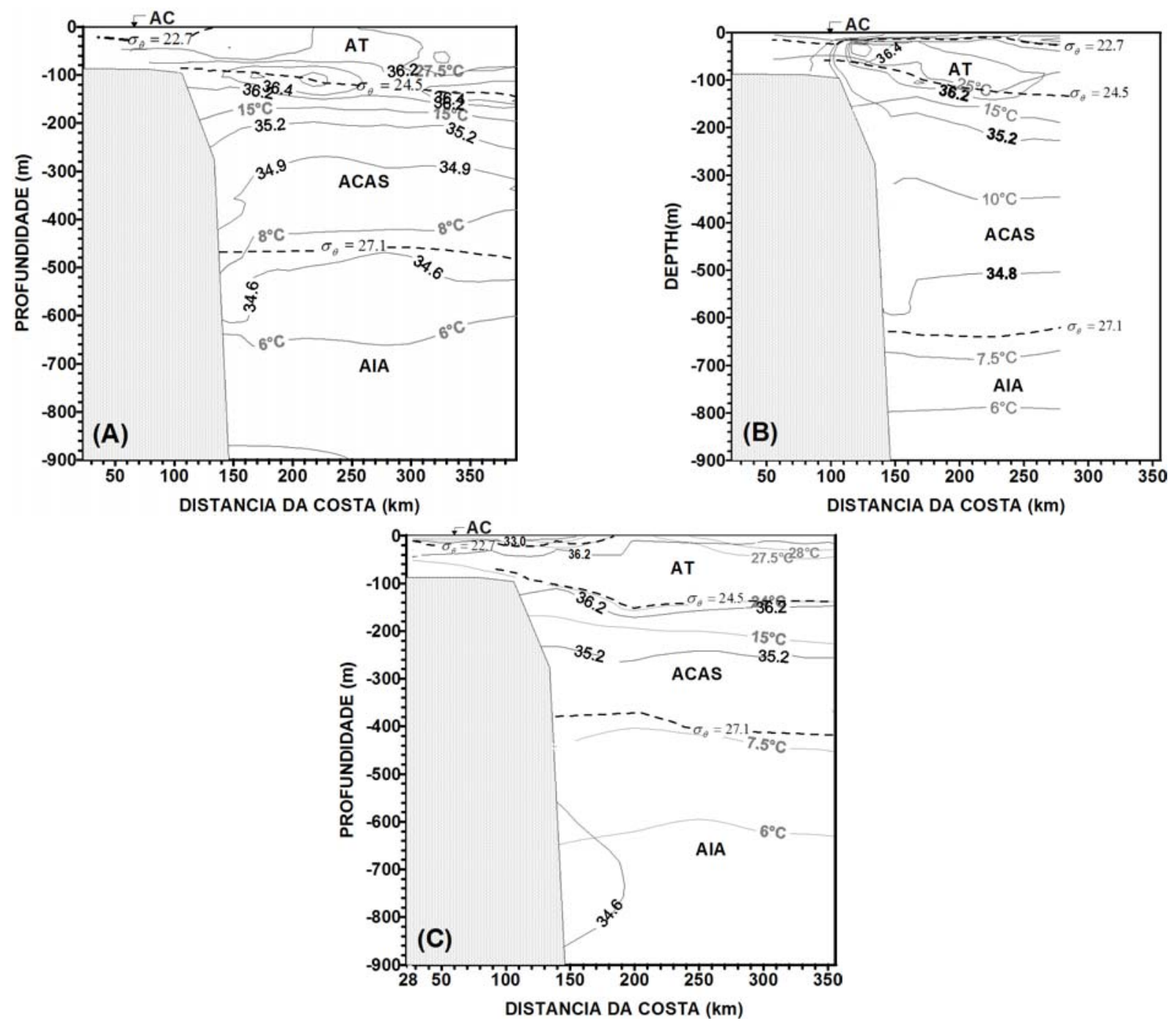

Figura 7 - Distribuição vertical das massas de água ao longo do transecto Perfil (W) durante: (A) período de descarga máxima do rio Amazonas (maio de 1999); (B) período de transição entre descarga máxima para mínima do rio (agosto de 2001); e (C) período de descarga mínima do rio Amazonas (outubro de 1997).

exclusivamente à região da PCA (limitada à isóbata 100 m), seja à região oceânica de profundidades superiores a $1000 \mathrm{~m}$.

As análises das observações sinópticas, obtidas através do Programa REVIZEE, mostraram que durante o período de máxima descarga (maio de 1999), a influência de uma pluma de água de baixo valor de salinidade provinda do rio Amazonas se estende oceano a dentro, muito além dos limites da PCA. Esta época coincide com o período em que se verifica o menor fluxo da CNB, paralelo à costa, com ventos alísios de nordeste incidindo quase que perpendicularmente às isóbatas. Estes fatores terminam por inibir o transporte para noroeste da pluma de água doce, o que foi claramente observado durante as épocas de máximo transporte da CNB, favorecido também pela ação dos alísios de su- deste (período de transição entre as vazões máxima e mínima do rio).

Os resultados aqui apresentados indicaram ainda que a distribuição espaço-temporal das massas d'água de subsuperfície (ACAS e AIA) pode ser qualitativamente explicada a partir da variabilidade sazonal do transporte da Corrente Norte do Brasil (CNB), de sua conexão com a Contra-corrente Norte Equatorial (CCNE), e da presença de vórtices sub-superficiais na região de retroflexão da CNB. Os transectos analisados mostraram que a ACAS sofre uma expansão vertical de cerca de $70 \mathrm{~m}$ durante o período de transição das descargas fluviais, quando comparada às demais épocas do ano (Perfil $\left.(\mathrm{A} / \mathrm{S}), 4^{\circ} \mathrm{W}\right)$. Nesta ocasião observou-se uma intrusão significativa das águas oceânicas 
(ACAS) sobre a PCA. Ao norte (Perfil (W), 50 $\mathrm{W}$ ), neste mesmo período do ano, esta expansão vertical é bem mais importante, chegando a atingir $220 \mathrm{~m}$ de coluna d'água. Estes cenários, que caracterizam uma expansão considerável do domínio das águas centrais no mês de agosto, coincidem com a ocorrência quase que simultânea dos picos do fluxo para noroeste da CNB, e de transporte de volume d'água retroflectado pela CCNE, favorecendo a formação vórtices anticiclônicos, sobretudo na região setentrional da área de estudo (Perfil (W), 50W).

Por fim, deve-se ressaltar que, embora em baixa latitude, o ajuste baroclínico da CNB também contribue para a intrusão (expansão) da ACAS no talude e PCA. Um interessante via para a continuidade deste trabalho nos parece associada à investigação da importância relativa destes processos (descarga fluvial, intensificação e ajuste baroclínico da CNB) sobre a variabilidade da estrutura de massas de água observada. Nesse sentido, a modelagem matemática da circulação oceânica na área de estudo aparece com uma ferramenta privilegiada para quantificação dos mecanismos envolvidos.

\section{AGRADECIMENTOS}

Os autores agradecem ao Dr. Maâmar El-Robrini, Coordenador do Sub-Comitê Norte do Programa REVIZEE, e sua equipe, pela disponibilização dos dados de CTD, assim como os Comandantes e tripulações do NOc. Antares, Marinha do Brasil, pelo esforço e dedicação demonstrados durante todas as expedições oceanográficas. 0 primeiro autor agradece à Coordenação de Aperfeiçoamento de Pessoal de Nível Superior - CAPES, pela concessão de Bolsa de Doutorado.

\section{REFERÊNCIAS}

BARNIER B, REYNAUD T, BECKMANN A, BONING C, MOLINES JM, BARNARD S \& JIA Y. 2001. On the seasonal variability and eddies in the North Brazil Current: insight from model intercomparison experiments. Progress in Oceanography, 44: 195-230.

BOURLĖS B, MOLINARI RL, JOHNS E, WILSON WD \& LEAMAN KD. 1999. Upper layer currents in the western tropical North Atlantic (19891991). Journal of Geophysical Research, 104(C1): 1361-1376.

BROWN WS, JOHNS WE, LEAMAN KD, MCCREARY RL, MOLINARI RL, RICHARDSON PL \& ROOTH C. 1992. A western tropical Atlantic experiment (WESTRAX). Oceanography, 5: 73-77.

CASTRO BM \& MIRANDA LB. 1998. Physical oceanography of the western Atlantic continental shelf located between $4^{\circ} \mathrm{N}$ and $34^{\circ} \mathrm{S}$. The Sea, 11: 209-251.
CURTIN TB. 1986. Physical observation of the plume region of the Amazon River during peak discharge. II. Water masses. Continental Shelf Research, 6: 53-71.

DHN. 1989. Diretoria de Hidrografia e Navegação. CXXXV Comissão Oceanográfica. Operaçãa Pavassas I. NOc. "Almirante Saldanha" (28/06 a 19/09/87). Marinha do Brasil, Rio de Janeiro, 28 p.

DIDDEN N \& SCHOTT FA. 1993. Eddies in the North Brazil Current retroflection region observed by Geosat altimetry. Journal of Geophysical Research, 98(11): 20,121-20,131.

FLOOD RD \& DAMUTH JE. 1987. Quantitative characteristics of sinuous distriburary channels on the Amazon. Bulletin of the American Geology Society, 98: 728-738.

FRATANTONI DM, JOHNS WE \& TOWNSEND TL. 1995. Rings of the North Brazil current: their structure and behavior inferred from observations and a numerical simulation. Journal of Geophysical Research, 100(6): 10633-10654.

GARZOLI SL \& KATZ E. 1983. The forced annual reversal of the Atlantic North Equatorial Countercurrent. Journal of Physical Oceanography, 13: 2082-2090.

GARZOLI SL, FFIELD A, JOHNS WE \& YAO Q. 2004. North Brazil Current retroflection and transports. Journal of Geophysical Research, 109, C01013, doi:10.1029/2003JC001775.

GEYER WR, BEARDSLEY RC, LENTZ SJ, CANDELA J, LIMEBURNER R, JOHNS WE, CASTRO BM \& SOARES ID. 1996. Physical oceanography of the Amazon Shelf. Continental Shelf Research, 16: 575-616.

GIBBS RJ. 1970. Circulation in the Amazon river estuary and adjacent Atlantic ocean. Journal of Marine Research, 28: 113-123.

JOHNS WE, LEE TN, SCHOTT FA, ZANTOPP RJ \& EVANS RH. 1990. The North Brazil Current retroflection: seasonal structure and eddy variability. Journal of Geophysical Research, 95(C12): 22103-22120.

JONHS WE, BEARDSLEY RC, CANDELA J, LIMEBURNER R \& CASTRO BM. 1998. Annual cycle and variability of the North Brazil Current. Journal of Physical Oceanography, 28: 103-128.

LENTZ SJ \& LIMEBURNER R. 1995. The Amazon River plume during AMASSEDS: Spatial characteristics and salinity variability. Journal of Geophysical Research, 100(C2): 2355-2376.

MULLER-KARGER FE, MCCLAIN CR \& RICHARDSON PL. 1988. The dispersal of the Amazon's water. Nature, 333: 56-69.

NITTROUER CA \& DEMASTER DJ. 1986. Sedimentary Process on the Amazon Continental Shelf: Past, Present and Future Research. Continental Shelf Research, 6: 5-50.

NITTROUER CA \& DEMASTER DJ. 1996. The Amazon shelf setting: tropical, energetic, and influenced by large river. Continental Shelf Research, 16: 553-574. 
OLTMAN RE. 1968. Reconaissance investigation of discharge and water quality of the Amazon River. US. Geological Survey Circular 552, Washigton DC. $16 \mathrm{p}$.

RICHARDSON PL, ARNAULT S, GARZOLI S \& BROWN WS. 1994. North Brazil Current retroflection eddies. Journal of Geophysical Research, 99: 997-1014.

SCHOTT FA, FISCHER J \& STRAMMA L. 1998. Transports and pathsways of the upper-layer circulation in the western tropical Atlantic. Journal of Physical Oceanography, 28: 1904-1928.
STRAMMA L \& SCHOTT F. 1999. The mean Flow Field of the tropical Atlantic Ocean. Deep Sea Research II, 46: 279-303.

UNESCO. 1981. Background papers and supporting data on the Practical Salinity Scale 1978, Technical Papers Marine Sc., 37: 144 pp.

WILSON WD, JOHNS E \& MOLINARI RL. 1994. Upper layer circulation in the western tropical North Atlantic Ocean during August 1989. Journal of Geophysical Research, 99(C11): 22513-22524.

\section{NOTAS SOBRE OS AUTORES}

Alex Costa da Silva. Geólogo pela Universidade Federal do Pará (UFPA) em 2000, atuando especificamente no Grupo de Estudos Marinhos e Costeiros da UFPA. Participou de Projetos como MADAM (Manejo e Dinâmica nas Áreas de Manguezais), e REVIZEE/SCORE-NO (Programa de Avaliação do Potencial Sustentável de Recursos Vivos na Zona Econômica Exclusiva/Sub-Comitê Região Norte), atuando neste último como coordenador da Subárea de Oceanografia Física. Doutorando em fase de conclusão do Laboratório de Oceanografia Física Estuarina e Costeira, Departamento de Oceanografia da UFPE (LOFEC/DOCEAN).

Moacyr Araújo. Engenheiro civil pela Universidade Federal de Pernambuco (UFPE), Mestre em Hidráulica e Saneamento pela Universidade de São Paulo (USP) e Doutor em Física e Química do Meio-ambiente pelo Institut National Polytechnique de Toulouse (INPT/França). Professor Adjunto do Departamento de Oceanografia da UFPE desde 1998, Iotado no Laboratório de Oceanografia Física Estuarina e Costeira (LOFEC), onde vem desenvolvendo atividades de ensino e pesquisas em Oceanografia Física costeira e oceânica. Vice-coordenador da Subárea de Oceanografia Física do Programa REVIZEE/SCORE-NE (Programa de Avaliação do Potencial Sustentável de Recursos Vivos na Zona Econômica Exclusiva/Sub-Comitê Região Nordeste).

Bernard Bourlès. Doutor em Oceanografia Física pela Université de Paris VI, com especialização na dinâmica do oceano Atlântico tropical. Pesquisador do Institut de Recherche pour le Devellopement- IRD/França desde 1997, participando ativamente em programas oceanográficos internacionais (STACS-WESTRAX, WOCE), e franceses (NOE, CITHER, ETAMBOT e EQUALANT). Responsável pela parte francesa do Projeto PIRATA (Pilot Research Moored Array in the Tropical Atlantic) desde 2002, e pelos programas EGGE (Estudo da Circulação Oceânica e de sua Variabilidade na Camada superior do Oceano Atlântico Tropical Leste) e AMMA (Análises Multidisciplinar da Mousson Africana). 